\title{
Competition and Selection Among Conventions
}

\author{
Rahmtin Rotabi \\ Cornell University \\ rahmtin@cs.cornell.edu \\ Cristian Danescu-Niculescu-Mizil \\ Cornell University \\ cristian@cs.cornell.edu \\ Jon Kleinberg \\ Cornell University \\ kleinber@cs.cornell.edu
}

\begin{abstract}
In many domains, a latent competition among different conventions determines which one will come to dominate. One sees such effects in the success of community jargon, of competing frames in political rhetoric, or of terminology in technical contexts. These effects have become widespread in the on-line domain, where the ease of information transmission makes them particularly forceful, and where the available data offers the potential to study competition among conventions at a fine-grained level.

In analyzing the dynamics of conventions over time, however, even with detailed on-line data, one encounters two significant challenges. First, as conventions evolve, the underlying substance of their meaning tends to change as well; and such substantive changes confound investigations of social effects. Second, the selection of a convention takes place through the complex interactions of individuals within a community, and contention between the users of competing conventions plays a key role in the convention's evolution. Any analysis of the overall dynamics must take place in the presence of these two issues.

In this work we study a setting in which we can cleanly track the competition among conventions while explicitly taking these sources of complexity into account. Our analysis is based on the spread of low-level authoring conventions in the e-print arXiv over 24 years and roughly a million posted papers: by tracking the spread of macros and other author-defined conventions, we are able to study conventions that vary even as the underlying meaning remains constant. We find that the interaction among co-authors over time plays a crucial role in the selection of conventions; the distinction between more and less experienced members of the community, and the distinction between conventions with visible versus invisible effects, are both central to the underlying processes. Through our analysis we make predictions at the population level about the ultimate success of different synonymous conventions over time - and at the individual level about the outcome of "fights" between people over convention choices.
\end{abstract}

(C) 2017 International World Wide Web Conference Committee (IW3C2), published under Creative Commons CC BY 4.0 License. WWW 2017, April 3-7, 2017, Perth, Australia. ACM 978-1-4503-4913-0/17/04. http://dx.doi.org/10.1145/3038912.3052652

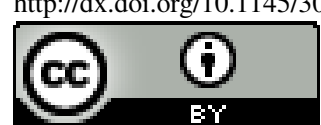

\section{Keywords}

conventions, innovations, diffusion of information, arXiv

\section{INTRODUCTION}

The diffusion of innovations, a rich area of study with its roots in sociology, has developed into a lens for addressing the spread of new ideas, information, behaviors, and technology across a wide range of domains 35. In recent years, the on-line domain has provided a powerful setting in which to study this process. The detailed view of the problem offered by such data has made it possible to provide new insights into many aspects of diffusion, including its temporal properties 10, its structural properties at both a local [4. 26, 44 and global level 2, 18, 27, its level of predictability 39, 9], and the characteristics of the participants [12, 38.

One of the central questions that theories of diffusion can address is the process of competition and selection among conventions: when there are multiple possible behaviors and a group must choose among them, can we characterize how this selection process takes place, and how the latent interaction between competing options unfolds? The rise of new idioms and terminology [12]; technical standards in engineering and technological domains 3 ; themes in political rhetoric [16]; and styles in artistic and other subjective domains 39 are all cases where we can pose such questions. It is important to note that the set of issues surrounding such conventions are far from monolithic - in particular, in cases with high costs to miscoordination, one tends to see a single convention crowd out all the others almost completely, while in cases where the convention has lower coordination effects and poses lower normative constraints, one typically finds extensive coexistence of conventions, with one convention dominating and others persisting in parts of the population 47.

Our work here begins by noting two central methodological challenges that arise in studying the evolution of conventions: one is an issue of content versus structure, and the other is an issue of local versus global effects.

- Substantive shift. First, it is possible for one convention to eclipse another because of a substantive shift - in which the substantive meaning of one convention has a relative advantage over the other. If we seek to understand the role that social structure plays, we must look for settings in which the competing conventions are essentially synonymous at the level of their substance. The work on this question to date has faced the challenge that in most natural settings it is hard to verify whether competing conventions are seman- 
tically equivalent, and thus to disentagle social effects from the relative advantage of one convention over another.

This is a distinction that is also familiar in studies of biological evolution, where the use of neutral variation - mutations that have minimal effect on an organisms's fitness has come to be an enormously influential methodology for studying effects of population structure on evolution in the absence of overt selective pressure 20]. What is the analogue of neutral variation in the diffusion of on-line information?

- Diffusion through interaction. Second, much of the work on diffusion - both theoretically and via on-line data - has studied the global competition among conventions via local mechanisms of contagion: these mechanisms posit that at a local level, the use of the convention spreads from one person to another, either probabilistically or through bestresponse behavior, and the competition among these contagion processes leads to the global outcome. But in most domains where non-trivial conventions are competing, the competition takes place not just globally but also locally through person-to-person interaction. Returning to our examples above: a single interaction between speakers in a dialogue or discussion, collaborators on a technical project, politicians framing a shared position, or artists performing a shared work may all implicitly involve competition between the conventions used by the participants in this interaction. The global outcome of the competition between two conventions may emerge from the results of thousands or millions of these micro-level competitions. This type of diffusion through interaction requires a fine-grained analysis of the local competition, rather than just a view of the local dynamics as concurrent contagion processes.

The present work. In this work, we propose an analysis framework for the competition and selection among conventions that explicitly addresses these issues of neutral variation and diffusion through interaction. We do this through a set of novel definitions and measures, together with a rich source of data that clearly display both notions at work.

Our data comes from a complete snapshot of the source files on the e-print arXiv, covering 24 years and over a million papers. We study how low-level authoring conventions emerge through the collaborations among different overlapping sets of co-authors over a multi-year time span. The source files on the arXiv provide a detailed view into a wide range of such authoring conventions; we focus primarily on the role of author-defined macros in $\mathrm{LTT}_{\mathrm{E} X}$ as one abundant supply of conventions. In writing the $\mathrm{LT}_{\mathrm{E}} \mathrm{X}$ source for a paper, authors will often define one or more macros as a way to make the writing of the paper easier and more modular; as in a standard programming language, each instance of a macro instance is specified by a name (henceforth name) and a body definition (henceforth body) which defines the functionality of the macro. Each time the formatting software for the paper sees the name of the macro, it replaces it with the body of the macro in the text; thus, for example, the command, \def $\backslash$ Reals $\{\backslash$ mathbb $\{R\}\}$ defines a macro, and whenever the author writes $\backslash$ Reals in the source file for the paper, the symbol $\mathbb{R}$ will appear in the outcome.

The appeal of focusing on macros is that they provide an extremely rich source of synonymous conventions in the social ecosystem of the arXiv. Whenever the name for a macro changes while the body remains the same - for example, when someone chooses to use $\backslash R$ instead of $\backslash$ Reals for the symbol $\mathbb{R}$ - the author is settling on an arbitrary choice of convention while the underlying meaning remains constant. As argued above, this type of control for meaning is crucial if we want to study the social structure around convention change separately from substantive shifts in content; however, controlling for meaning is very hard to achieve unless one has an almost mechanistic specification of this meaning. Macros provide us with precisely such a specification in their body. Also, they are pervasive in arXiv: roughly $40 \%$ of all arXiv papers contain at least one user-defined macro.

Moreover, because papers on the arXiv are largely coauthored, the competition among synonymous macros is also a powerful setting in which to define and then study some of the basic properties of diffusion through interaction. Two macro names with the same body are competing not just globally based on their relative prevalence in the full population of papers, but also locally each time two people who follow different conventions come together to co-author a paper. Analyzing the history of the arXiv provides us with a way to study how such instance-by-instance competition plays out in the context of these larger diffusion processes.

The arXiv thus provides us with the ingredients for analyzing information diffusion in a way that addresses these methodological challenges. At the same time, we note that the arXiv is of course a controlled domain representing a single type of broad activity — scientific authorship — and as such our work is approaching these issues via a case study of this particular domain. It will be interesting to study how the observations here generalize to different contexts; our approach is set up to facilitate this by providing a road map for these types of analyses across domains.

Overview of results. We begin by using the controlled setting provided by our data to study the competition between synonymous conventions at a global level. A concrete way to formulate this question is to look at two competing names for the same macro body up to a certain point in time, and ask whether we can predict which name will become dominant at some point in the future. First, we find that properties of the name itself - e.g., features related to its orthography, since the meaning is fixed - do not seem to have any predictive power; the differences in the competing names for the same macro body appear to truly represent neutral variation, a fact that offers a striking opportunity to explore other features in the absence of selective pressure ${ }^{1}$

We find, instead, that features related to the experience of a name's early users - the number of previous papers that each has written on the arXiv - have significant predictive value for the question of whether a macro name will grow to become dominant. In general, names that eventually become dominant tend to start with an initial author population that is relatively "younger" (with lower experience), and then they successfully spread to "older" users. Names that don't achieve dominance are more associated with initial user populations that are older in aggregate, and also fail to spread to new adopters with higher experience. These hand-offs between different "generations" of people, and how

\footnotetext{
${ }^{1}$ The fact that the name itself provides no predictive power may be in part a reflection of the fact that authors tend to choose reasonable and informative names for their macros; it is easy to imagine that a particularly inapt name could have more difficulties in its adoption, but this is not the situation that generally seems to apply.
} 
they contribute to the success of a convention, is an interesting issue connected to the role of status in diffusion [12, 35, and the results from our data suggest interesting directions in which to explore these issues further.

We next ask how this competition plays out at a local level, dropping down from the global scale in order to explore diffusion through interaction. We develop a framework for analyzing the instance-by-instance competition that arises when authors following different synonymous conventions meet to collaborate. In particular, if authors $A$ and $B$ meet for the first time to write a two-authored paper, and they have previously used different macro names for the same macro body, how does the resulting "fight" over the choice of convention turn out? We find that the relative experience level of the two authors is again a highly informative property of the interaction; the author who is "younger" (with lower experience) tends to win these fights, with the probability of winning increasing as the gap in experience grows. Building a set of features based on experience - both numerically and through certain more complex structural analogues, such as the authors' graph-theoretic properties in the larger co-author network - we are able to develop methods for predicting the outcomes of these fights with non-trivial accuracy.

It is an interesting question to consider possible mechanisms for the dominance of younger participants in these instance-by-instance fights; a natural hypothesis is that they play a larger role in the detailed implementation of the paper, and hence have more control over definitional questions such as macros. Such a model would suggest the conjecture that younger co-authors should not necessarily win fights over questions that are less about low-level implementation and more about high-level, visible decisions where the status of the older co-author is arguably more implicated. We show that this indeed appears to be the case, by studying the latent competition between co-authors over conventions in the title of the paper, rather than the macros. We can think of the title as occupying the opposite end of the visibility spectrum from macro names, in that decisions about titling conventions are highly visible; and here, under a set of definitions that we formulate in the paper, we find that the older co-author tends to win fights about titling conventions, with the effect increasing as the experience gap increases (here in the opposite direction from what we saw in fights over macro names). In summary, our results suggest the beginnings of a set of principles that could be summarized in caricature as, "In a collaboration, the younger people win the invisible fights while the older people win the visible fights." We argue that developing this notion more deeply is an interesting direction for further research, and we point to some additional steps along these lines.

In summary, our focus is on developing new definitions around some fundamental issues that have been difficult to address in diffusion and the selection of conventions - the role of neutral variation, represented through the properties of synonymous conventions, and the dynamics of competition not just at a global level but through the continuous low-level competition between users of different conventions as they interact in the system. We hope that our exploration of these definitions and concepts in a case study on the arXiv will indicate how such analyses can be carried more broadly across other domains as well.

\section{FURTHER RELATED WORK}

Words as conventions. One of the most widespread sources of conventions is in the choice of words used to refer to particular concepts. As noted above, our use of macros is designed to contrast with an inherent source of complexity in the analysis of conventions in natural language, namely the severe difficulty in controlling for the precise meaning of a concept as the words referring to it change.

Analysis of changes in language over long time periods has considered a dual problem to ours: how fixed linguistic constructs acquire new meanings. This has been undertaken recently in studies of historical shifts in word meanings 41 19] and grammatical constructions 33], relying on books and news data that span long periods of time. Related studies have been performed in the on-line domain, analyzing global changes in the linguistic system of Twitter 15, 14, 17] and other on-line communities 12,25 .

Sociolinguistic studies of linguistic change have addressed changes in phonology and spelling that vary systematically across time 23, status 24] and region 32. As such, these studies are similar to ours in that they also explore variations in form of conventions used to refer to fixed concepts (e.g., whether the final ' $r$ ' in 'car' is pronounced or not), albeit the discussions are generally limited to a handful of examples.

Diffusion of information and cultural items. As discussed in the introduction, there has been a long line of work studying the processes by which discrete units of information diffuse on-line; these include memes [28, 31], hashtags on Twitter [36, 37, 29] and on-line news content [1, 7, 5]. A growing strand of research within this topic has considered the problem of predicting future popularity, with specific prediction studies involving downloadable content 39], quotes embedded in broader cultural contexts [11, 6], hashtags 42, and memes 9,43 .

Most of these previous studies could not control for the meaning of the convention or content that is spreading, with two notable exceptions. The first is a study on the emergence of the retweet convention on Twitter 21]; this represents an in-depth study of a single convention providing extended insights, in contrast to our study of thousands of distinct conventions and the common properties across them. The second is a study of competition between hashtags on Twitter that only differ in capitalization, suffixes, or relative levels of abbreviation (e.g., \#saveTheNationalHealthService vs. \#savethenationalhealthservice vs. \#savetheNHS) 43. Our setting allows for a more general way of identifying synonymous conventions, and for verifying that they are indeed synonymous. And perhaps more crucially, the study of hashtags in 43 showed that the orthography of different hashtags was in fact predictive of their success, establishing that in fact these different versions of hashtags do not represent neutral variations as in our case, but instead variation that affects relative fitness.

Role of experience. Our work also explores the interplay between individuals' levels of experience and their roles in the diffusion of conventions, including the question of whether new conventions originate with younger members of the community, or whether the older members have a relative advantage in imposing their forms of conventions. Such 
questions about trade-offs based on experience and status in the diffusion of innovations has a long history of study in off-line domains $13,34,40,30,45,8,22$, and more recently has been explored in on-line domains as well 12,38 . However, these lines of work do not look at instances where synonymous conventions compete with each other, or where it is possible to see such competition playing out at a local level through person-to-person contention.

\section{DATA}

As discussed in the introduction, we use a dataset of macros from the e-print arXiv, so as to be able to look at variations in language conventions (the names of macros) while controlling for their meaning (the body).

The arXiv is a repository of scientific pre-prints (covering physics, mathematics, computer science, and an expanding set of other scientific fields). The arXiv contains the source files for almost all the papers that have been uploaded to it, with most of these written in $\mathrm{AT}_{\mathrm{EX}}$. Our dataset consists of the full source of all $\mathrm{LTT}_{\mathrm{EX}}$ files on the arXiv, from its inception (July 1991) through September 2015, a corpus of over a million papers. For a prefix of this time period, the ordering of the papers in our data is only resolved up to one-month granularity (the remainder is totally ordered), but our methods work with this level of granularity.

From the IATEX source files we extract all macros defined by the most common methods, specifically \def, \newcommand and \renewcommand. This results in macros from over 400, 000 papers. Note that we do not recursively substitute names that occur inside of another macro body. Table 1 summarizes basic statistics about our data $2^{2}$

Table 1: Dataset details

\begin{tabular}{lr}
\hline Number of papers with a macro & 583,078 \\
Number of macros defined & $22,628,300$ \\
Number of unique macro bodies & $2,586,548$ \\
Average number of names per body & 1.40 \\
Number of unique authors & 222,689 \\
Average author per paper & 2.35 \\
\hline
\end{tabular}

\section{GLOBAL COMPETITION BETWEEN CONVENTIONS}

We begin by considering the competition between conventions, in the form of macro names, at a global level. To give a concrete sense for the behavior we are interested in studying, here is a simple example of competition among macro names - one of many with a similar flavor on the arXiv. In March 1996, Luty, Schmaltz, and Terning posted a paper to the arXiv, on an application of gauge theories in theoretical physics, in which they defined the macro name \Yfund to expand to a macro body representing a very simple instance of a combinatorial structure known as a Young tableau:

\section{$\backslash$ raisebox $\{-.5 p t\}\{\backslash$ drawsquare $\{6.5\}\{0.4\}\}$}

This macro body was used again (with the same name \Yfund) in two more papers in May 1996, seven more in the remainder of 1996, and a steady stream of others after that. Of

${ }^{2}$ Our macro dataset is available at http://github.com/ CornellNLP/Macros; a repository of arXiv papers is avallable at http://arxiv.org/help/bulk_data_s3. the first 42 uses of this macro body, all but two referred to it by the name \Yfund. (The other two used \fun, a name that never really caught on.) But then, in a paper in May 1998, Hanany, Strassler, and Uranga used the name \fund to refer to this macro body. A competition soon broke out between \Yfund and \fund, with \fund gradually becoming more prevalent. The macro body has by now appeared in over 750 papers on the arXiv; of the most recent 100 uses, 39 used \Yfund and 61 used \fund. Figure 1 shows how this changeover between the two macro names took place; the $x$-axis is a time axis, indexed in order of uses of the macro body, and the $y$-axis shows a sliding-window average of the fraction of authors using the names \Yfund and \fund as a function of time.

This type of dynamic has played out with many macros on the arXiv, and the point is not that the choice of macro name is consequential for the substance of the authors' research. In fact, the point is the opposite: changes in the macro name are a source of neutral variation, essentially incidental to the real progress of the community, and hence they let us probe the changeover dynamics in conventions at the level of individuals, their characteristics, and their interactions.

We also note that the competition underlying the changeover dynamics can take several different possible forms. It may be that authors following the two conventions interact directly through co-authorship or other mechanisms. But it may also be that one convention overtakes another even without direct interaction between the followers of the two conventions, simply because one of the two conventions grows in adopters and usage significantly faster than the other. This too is a form of competition between the conventions, played out in their relative rates of growth.

Defining changeovers. We now describe how we identify a broad set of instances in which one macro name overtakes another. For parameters $s, q$, and $\theta$, we find macro bodies that have at least $s$ total occurrences, where there is a name $N_{e}$ that is the most used name in the first $q$ fraction of occurrences, and a different name $N_{\ell}$ is the most used in the last $q$ fraction of occurrences. Moreover, each of $N_{e}$ and $N_{\ell}$ is widely used in the sense that $N_{e}$ is used by more than a $\theta$ fraction of authors who use the macro body in its first $q$ fraction of occurrences, and that $N_{\ell}$ is used by more than a $\theta$ fraction of authors who use the macro body in its last $q$ fraction of occurrences. In our analysis, we use $s=100, q=0.3$ and $\theta=0.3$, although other choices of these parameters produce similar results.

If these properties are met for a given macro body $\beta$, we say that $\beta$ undergoes a changeover from $N_{e}$ to $N_{\ell}$, and we refer to $N_{e}$ as the early name for $\beta$, and $N_{\ell}$ as the late name for $\beta$. In Figure 2 and 3 we show some aggregate properties of the set of changeovers on the arXiv. First, consider a given macro body $\beta$ with $m_{\beta}$ occurrences; for any $0 \leq t_{0} \leq t_{1} \leq 1$, we define the interval $\left[t_{0}, t_{1}\right]$ in the body's lifespan to be the set of papers indexed between $t_{0} m_{\beta}$ and $t_{1} m_{\beta}$ in the time-sorted ordering of papers using $\beta$. We will refer to quantities like $t_{0}$ and $t_{1}$ as "times," (or "macro lifestages") corresponding to a fraction of the way through a macro body's lifespan on the arXiv. Now, if $\beta$ undergoes a changeover, we define the function $f_{\beta}\left(t, t^{\prime}\right)$ to be the fraction of authors in the interval $\left[t, t^{\prime}\right]$ that use the early name $N_{e}$, and we define the function $g_{\beta}\left(t, t^{\prime}\right)$ to be the fraction of authors in the interval $\left[t, t^{\prime}\right]$ that use the late name $N_{\ell}$. We 


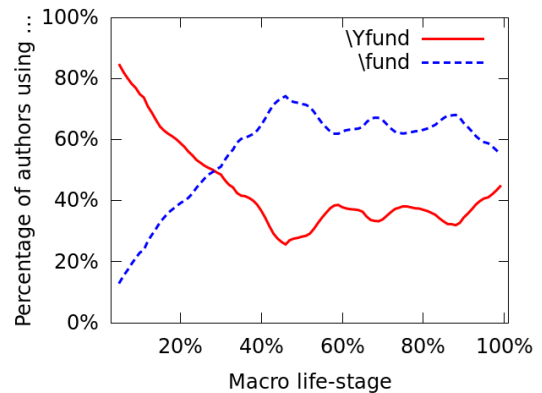

(a) Example changeover

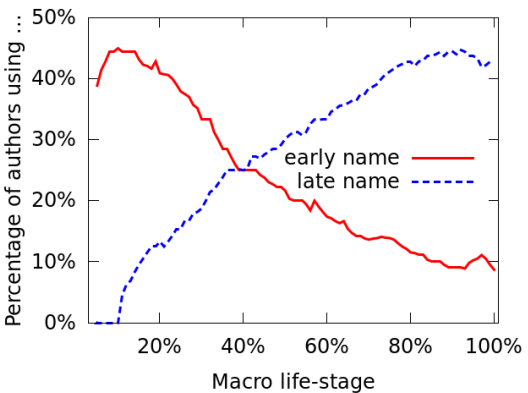

(b) All changeovers aggregated

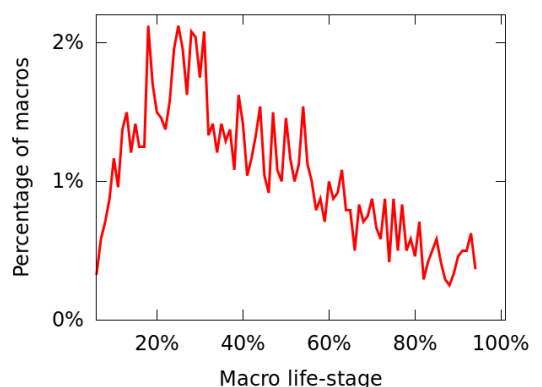

(c) Crossing-point distribution

Figure 1: Changeover in conventions. (a) An example changeover: \fund surpasses the once-dominant \Yfund as the preferred name used to invoke Young tableau; y-axis indicates the percentage of users of each name out of all authors using the respective body. (b) Aggregated temporal usage trends of early name ( $\left.N_{e}\right)$ and late name $\left(N_{\ell}\right)$ for all macros undergoing changeovers; their crossing point is well before the middle of their lifespan. (c) The distribution of crossing points (percentage out of all macros undergoing changeovers).

can turn these into single-variable functions by fixing an increment $\delta$ and defining $f_{\beta, \delta}(t)=f_{\beta}(t, t+\delta)$ and $g_{\beta, \delta}(t)=$ $g_{\beta}(t, t+\delta)$; these are just the fractions of usage in length- $\delta$ intervals beginning at $t$.

Figure 2 fixes $\delta=0.05$ and shows the median values of $f_{\beta, \delta}(t)$ and $g_{\beta, \delta}(t)$, as functions of $t$, aggregated over all $\beta$ that undergo changeovers. It is intuitively sensible that $f_{\beta, \delta}(t)$ should be falling in $t$ and $g_{\beta, \delta}(t)$ should be rising in $t$, since $N_{\ell}$ is in effect partially taking over from $N_{e}$. It is intriguing, however, that the shapes of the two curves are not symmetric in time - in that they cross well before the midway point at $t=0.5$ - considering that the definition of changeover is temporally symmetrical.

Figure 3 shows the distribution of these crossing points, over all $\beta$ that undergo changeovers. For this plot, we formalize the crossing point as the minimum $t$ such that $g_{\beta, \delta}\left(t^{\prime}\right) \geq$ $f_{\beta, \delta}\left(t^{\prime}\right)$ for all $t^{\prime} \in[t, t+.1]$, so as to require that the crossing persist for a non-trivial interval of time. This plot too highlights the fact that the crossing tends to occur early in the usage of the macro body $\beta$, well before the midway point, although there is considerable diversity - for some macro bodies, the crossing point comes very late.

\subsection{Properties of the authors in a changeover}

We now examine the properties of the authors who use competing names in a changeover. In order to have a baseline for comparison, we pair macros undergoing changeovers with macros that do not undergo changeovers, but which have similar behavior up to their first $q$ fraction of uses.

Thus, for each macro body $\beta$ that undergoes a changeover, we find a macro body $\gamma$ that does not undergo a changeover, for which (i) the total volumes of usage are approximately the same, $m_{\beta} \approx m_{\gamma}$, and (ii) there are two names $N_{e}^{\prime}$ and $N_{\ell}^{\prime}$ for $\gamma$ such that the prevalence of these two names in their early phases are approximately the same as $N_{e}$ and $N_{\ell}$ respectively: $f_{\beta}(0, q) \approx f_{\gamma}(0, q)$ and $g_{\beta}(0, q) \approx g_{\gamma}(0, q){ }^{3}$ We will refer to $\beta$ and $\gamma$ as a matched pair of macro bodies. Intuitively, from the perspective of their volume up to time $q$, the two names $N_{\ell}$ and $N_{\ell}^{\prime}$ - for $\beta$ and $\gamma$ respectively -

\footnotetext{
${ }^{3}$ The precise filter we use is to require that $m_{\beta} / m_{\gamma} \in$ $[.91,1.1]$ and $\left|f_{\beta}(0, q)-f_{\gamma}(0, q)\right|$ and $\left|g_{\beta}(0, q)-g_{\gamma}(0, q)\right|$ are both below .01.
}

had very similar initial conditions, and hence we are forced to look at other properties to find a difference between them.

A key property that we consider is the experience of the authors using these names; recall that an author's experience at a given point in time is the number of papers they've written up to that time, which measures a kind of "age". (Accordingly, when we refer to authors as "younger" or "older," it is with respect to this measure of experience, and not to biological age.) Now, for a matched pair of macro bodies $\beta$ and $\gamma$, we can look at the following quantities at a time $t \in[0,1]$ : the average usage experience of authors of each given name at time $t$, as well as the average adoption experience of users of each given name at time $t$; the former quantity aggregates over the experience of all users of the given name at time $t$, while the latter quantity aggregates only over the experience of authors using the given name for the first time at $t$.

In Figure 2 we show the average usage experience (left panel) and average adoption experience (right panel) for the four names $N_{e}, N_{\ell}, N_{e}^{\prime}$, and $N_{\ell}^{\prime}$, averaged over all matched pairs $(\beta, \gamma)$. We notice a few respects in which these curves exhibit similar properties between $\beta$ and $\gamma$ : first, they all increase, which is natural since experience values are increasing as time runs forward on the arXiv. Moreover, the curves for $N_{\ell}$ and $N_{\ell}^{\prime}$ start out below the curves for $N_{e}$ and $N_{e}^{\prime}$, which is consistent with the intuition that new terminology tends to start with more peripheral authors 38.

However, the curves for $\beta$ and $\gamma$ also differ in important ways, and this provides us with some insight into the differences between macro bodies $\beta$ that undergo changeovers and macro bodies $\gamma$ that don't. First, and most visibly, the name $N_{\ell}$ performs a major transition over its lifetime, going from authors with very low experience to authors with very high experience, while the experience of authors using $N_{e}$ plateaus. Conversely, $N_{\ell}^{\prime}$ fails to perform a corresponding transition, and remains concentrated on authors of low experience throughout its lifespan. In this sense, $N_{\ell}$ and $N_{e}$ almost "change roles" as the plot progresses, with the curve for $N_{\ell}$ initially tracking $N_{\ell}^{\prime}$ but eventually tracking $N_{e}^{\prime}$, and the opposite holding for $N_{e}$. There is also a small but significant difference at the smallest values of $t$ : the average experience for $N_{\ell}$ starts out lower than for $N_{\ell}^{\prime}$, a difference 


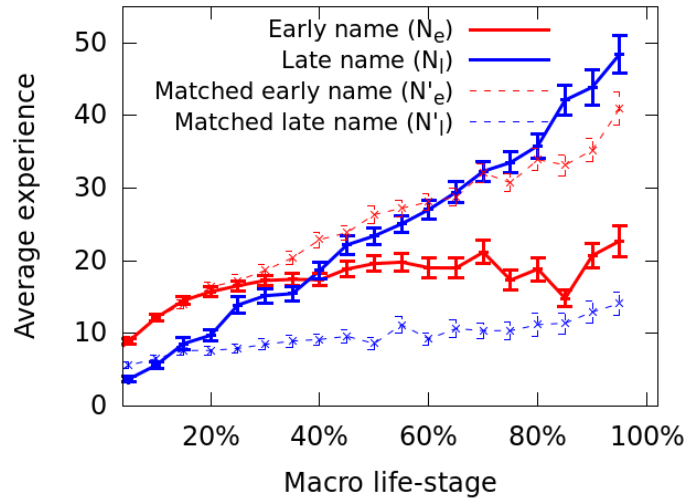

(a) Average usage experience

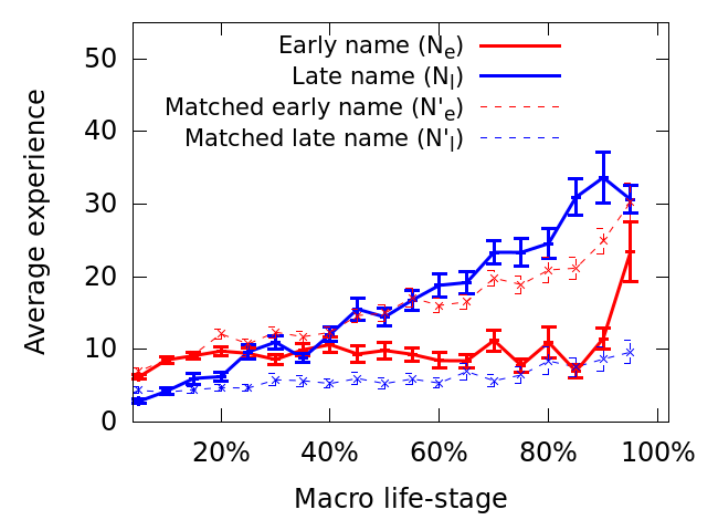

(b) Average adoption experience

Figure 2: Changeovers and user experience. When comparing names that eventually overtake their competitors ( $N_{l}$, solid blue lines) with those that don't ( $N_{l}^{\prime}$, dashed blue lines), we observe that they tend to start with a younger user-base and then successfully transition to more experienced users.

that may point to the value of low author experience in predicting the eventual success of a macro name. (We explore this further when we look at interaction dynamics in the next section.)

\subsection{Predicting changeovers}

We can also evaluate whether the properties we have assembled about the authors using competing names hold predictive power in the task of forecasting whether a changeover will occur. We formulate this as a prediction task, where for each matched pair of macro bodies $\beta$ and $\gamma$ - each involving two competing names - we try to predict early on which of them will undergo a changeover. Notice that because of the matching process, we have a balanced dataset where the two classes have the same aggregate characteristics in the early stages of their lifespans.

We first find that using only properties of the two competing macro names themselves - length, number of nonalphabetic characters, proportions of lowercase and uppercase characters - provides no predictive power. In other words, we can't predict simply from names like \Yfund and $\backslash$ fund which one will prevail. This reinforces the sense in which these truly represent neutral variations; the changes in name do not seem 4 to be fitness-enhancing on their own.

However, we get non-trivial predictive power when we add attributes of the authors using the names in their early stages. In particular, we define features based on the number of distinct authors using each name in the first $q(=0.3)$ fraction of the macro body's lifespan, as well as the average usage experience and average adoption experience in windows of $[t, t+.05]$ for $t \in\{0, .05, .10, .15, .20, .25\}$.

Here and in the rest of the paper, we perform logistic regression using features that are normalized using the $\mathrm{z}$ score, with data that has balanced labels and $80 / 20$ split on data for training and testing. The accuracies using different subsets of the features are presented in Table 2 The most important features are the average usage experience and the average adoption experience, with low values favor-

\footnotetext{
${ }^{4}$ There is, however, the possibility that more complex name features could turn out to hold predictive power.
}

ing changeovers: a name used by a younger generation is more likely to take over its competitor.

Table 2: Accuracy of changeover prediction ( $\pm 4 \%$ confidence intervals for all rows).

\begin{tabular}{lc}
\hline Feature set & Accuracy \\
\hline Random baseline & $50 \%$ \\
Name features & $50 \%$ \\
Average usage experience features & $58 \%$ \\
Average adoption experience features & $60 \%$ \\
All author-based features & $59 \%$ \\
All features & $57 \%$ \\
\hline
\end{tabular}

\section{DIFFUSION THROUGH INTERACTION: DYNAMICS OF LOCAL COMPETITION}

We now begin with a set of analyses designed to study how diffusion through interaction is taking place in our domain - the way in which competition over a set of conventions, in the form of different macro names for the same macro body, is taking place at a paper-by-paper level.

The "age" of the authors will again play an important role in these analyses, and we continue to use the experience of an author - the number of papers they have written - as a measure of age. Unless otherwise specified, when we are considering an author in the context of a particular paper they have written, we will be thinking about their experience at the moment this paper was written (as opposed to their eventual experience at the end of our dataset).

\subsection{Fights over macro names}

We now consider the outcome of competition between two authors who have recently used different conventions for the same macro body. In order to have a consistent structured setting for such competition, we consider situations in which two authors $A$ and $B$ meet to write a paper, each having used a different name for the macro body $\beta$, and one of these two names is used in their co-authored paper. In this case, 


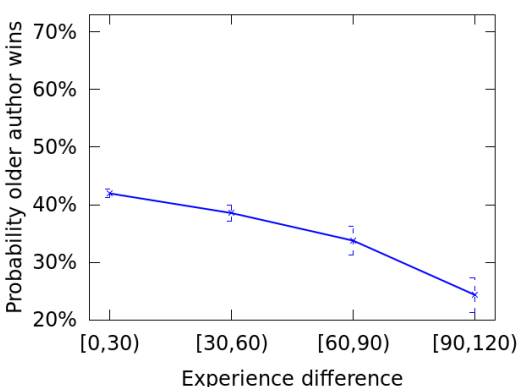

(a) Invisible fights: name

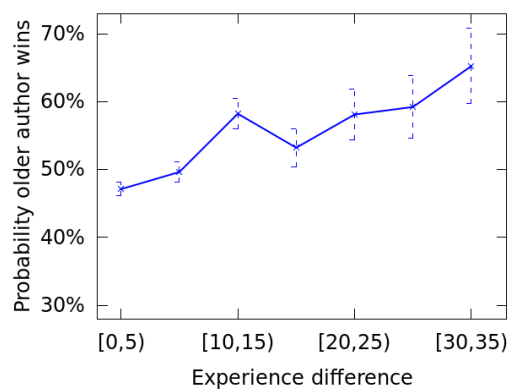

(b) Visible fights: paper title

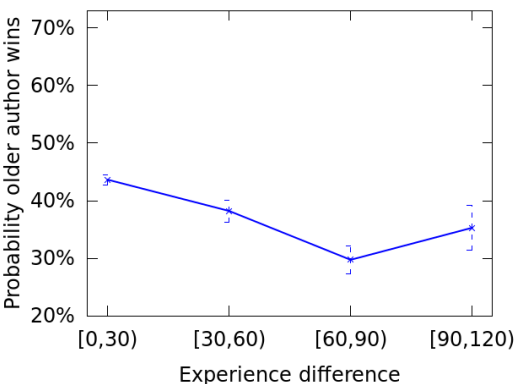

(c) Low-visibility fights: body

Figure 3: Percentage of fights won by the older author as a function of difference in experience. The larger the experience gap, the more likely the younger author is to win the (invisible) macro name fights (a) and the (low-visibility) macro body fights (c); the opposite trend holds for the (much more visible) title fights (b).

we say that a fight has occurred between $A$ and $B$ over the choice of name for body $\beta$, and the fight is won by the author whose name is used in their joint paper. A basic question is to characterize and potentially predict the winner of a fight of this form - how does it depend on the properties of $A$ and $B$, and potentially of the macro body and name?

More formally, we require that (i) two authors $A$ and $B$ write a paper $P$ in which they are the only co-authors ${ }^{5}$ (ii) the paper $P$ involves a macro body $\beta$ that each author has used before; (iii) in their most recent uses of $\beta$ (in earlier papers), $A$ and $B$ used names $\eta_{A}$ and $\eta_{B}$ with $\eta_{A} \neq \eta_{B}$; and (iv) one of $\eta_{A}$ or $\eta_{B}$ is used as the name for $\beta$ in the new co-authored paper $P$. Further, in order to study macros that have non-trivial usage, we require that the macro body be used in the dataset by at least 30 distinct authors, and that the length of the body be at least 10 characters. Also, since certain pairs of authors might satisfy conditions (i)(iv) many times, and there might be close alignment in the outcome of all their fights, we only consider a single fight for all pairs of papers that $A$ and $B$ co-author, selecting a chronologically earliest one ${ }^{6}$

The left panel of Figure 3 shows that the younger author (the one with lower experience) wins these fights significantly more often than the older author, with the probability that the older wins decreasing as the experience difference between the two authors increases. These results are significant $(p<0.05)]^{7}$ using the full set of 1574 fight instances that passed the conjunction of all the filters described above, and was balanced to control for author-position effects, with the first and second authors winning an equal number of fights.

A prediction task. We can use this distinction in experience to perform a prediction task: given an instance of a fight, and the past history leading up to it, how accurately can we predict who will win the fight?

\footnotetext{
${ }^{5}$ For robustness, we also redo the analysis on three-author papers, examining fights between the second and the third authors and obtain qualitatively similar results.

${ }^{6}$ For the portion of our data in which we only have time granularity at the one-month level, we further restrict to instances in which $A$ and $B$ have no other papers in the month of their current co-authorship and their previous usage of $\beta$, making the temporal ordering unambiguous.

${ }^{7}$ Here and throughout the paper we use the binomial test for statistical significance.
}

The most basic approach to this would be to simply formulate a prediction task problem using two features: the experience of the first author and the experience of the second author, aiming to guess which of them will win the fights. The left panel of Figure 3 suggests that we would already be able to achieve non-trivial performance from just these two features, and we find that we achieve $58.2 \%$ accuracy using a logistic regression model. If we declare an instance to have label 0 when the first-listed author wins the fight, and label 1 when the second-listed author wins the fight, then we can interpret the coefficients for the experience of these two authors in the logistic regression model, shown in Table 3 Note that the positive coefficient for the first author (Experience 1) means that higher first-author experience produces an output of the logistic function that favors label 1, corresponding to the second author winning the fight. Conversely, the negative coefficient for the second author (Experience 2) means that higher second-author experience favors the label 0 , corresponding to the first author winning. In consequence, for both authors higher experience works against them winning the fight, and lower experience tends to favor them in the prediction.

Table 3: Feature coefficients for predicting macro fights outcome when only using experience.

\begin{tabular}{ccc}
\hline & Experience 1 & Experience 2 \\
Feature coefficient & 0.40 & -0.59 \\
\hline
\end{tabular}

We can achieve non-trivially higher prediction performance by including a set of other natural features about the instance as follows. In particular, we use the following set of features.

- Experience: The number of papers the author has written prior to the fight.

- Prior uses: The number of prior papers in which the author has used the macro body.

- Flexibility: The fraction of consecutive uses of the body in which the author used two different names. (This is a measure of "flexibility" in that it shows the fraction of prior uses of the body in which the author changed names relative to their immediately preceding use.) 
Table 4: Top 6 feature coefficients for predicting the outcome of macro fights.

\begin{tabular}{lcccccc}
\hline & Experience 1 & Experience 2 & Flexibility 1 & Flexibility 2 & Degree 2 & Betweenness 2 \\
Feature coefficient & 0.48 & -0.54 & 0.47 & -0.54 & -0.38 & 0.55 \\
\hline
\end{tabular}

- Degree: We build a co-author graph on all authors who have used body, based only on papers that were written prior to the fight. This feature is the degree of the author in this co-author graph.

- Betweenness: The betweenness of the author in the co-author graph from the preceding point. (This is one measure of how central the author is in the graph.)

- Properties of name: The length of name.

- Properties of body: The length, number of nonalphabetic characters and the maximum depth of curly braces in body.

When we put all these features together, we are able to obtain $67.3 \%$ accuracy via logistic regression. The coefficients with the 6 largest absolute values in the model are shown in Table 4 Experience behaves as before; degree is naturally aligned with experience (since older authors have more time to acquire co-authors); and flexibility behaves as one would intuitively expect (since more flexible authors are more likely to also change in the current fight). It is interesting that high betweenness helps predict that an author will win a fight, since the results on experience might have suggested the opposite intuition.

To summarize, as noted in the introduction, it is interesting that the older author does not play the dominant role - relative to the younger author - in determining the outcome of the convention, given what we know about the tendency of high-status individuals to drive the outcomes of interactions [46]. A natural hypothesis is that the older author is ceding control over low-level decisions on conventions like macro names to the younger author. This suggests that we may arguably see a different outcome if we were to look at fights over decisions that were less low-level, more visible to readers, and hence more prominent. In such a case, where the status of the older author is more implicated by the outcome in the eyes of readers, is the older author more likely to win the fight? We now describe an analysis that addresses this contrast.

\subsection{Visible fights}

What would a more visible type of fight look like, and how does the young-old dynamic work in this case? We now formalize a set of fights involving one of the most visible decisions about a paper — the choice of title. For fights involving titles, we need a different set-up than the one we used for macros, and a more indirect one. In the case of macros, the convention was the choice of a name for a macro body, but since paper titles are generally written in a freeform manner, we need to decide what the space of possible conventions is.

In order to have a concrete definition to work with, and one that intuitively has a visible effect on the style of the title, we define conventions in the choice of title based on the presence or absence of certain punctuation, formatting, or parts of speech. Specifically, for a given title, we ask whether it exhibits one of seven possible styles (not all mutually exclusive): Does it contain a colon, question mark, or mathematical notation; and is its first word a noun, verb, adjective, or determiner? For each of these seven questions, we say that a title is a positive instance of the corresponding style if it contains the indicated feature.

Now, if authors $A$ and $B$ write a paper together, how do we define the notion of a fight over one of these stylistic titling conventions? Asking about the immediately preceding title for each author produces data that is too sparse to get meaningful results, and so instead, we look at each author's lifetime tendency to use each of the stylistic conventions.

Defining a fight over the title. Specifically, let us fix one of the stylistic conventions $\sigma$ defined above, and consider a two-authored paper in which the authors have never written a paper before ${ }^{8}$ Let $E_{y}$ and $E_{o}$ denote the experience of the younger and older authors on this paper respectively, and let $P_{y}$ and $P_{o}$ be the lifetime fraction of papers on which the younger and older authors used convention $\sigma$, only considering papers they did not write together ${ }^{9}$ Finally, we let $I_{\sigma}$ be an indicator variable equal to 1 or 0 depending on the presence or absence of the convention in the paper.

Intuitively, we'd like to consider the value of $I_{\sigma}$ in relation to which of $P_{y}$ or $P_{o}$ is larger. To have a meaningful baseline for comparison, we match each of our fights in pairs: for each fight given by $\left(E_{y}, E_{o}, P_{y}, P_{o}, I_{\sigma}\right)$, we find a fight using the same $\sigma$ but a different paper, where the values of $P_{y}$ and $P_{o}$ are swapped, and where $I_{\sigma}$ is inverted. That is, we find a fight $\left(E_{y}^{\prime}, E_{o}^{\prime}, P_{y}^{\prime}, P_{o}^{\prime}, I_{\sigma}^{\prime}\right)$ with $P_{y}^{\prime} \approx P_{o}$, and $P_{o}^{\prime} \approx P_{y}$, and $I_{\sigma}^{\prime}=1-I_{\sigma}$. Thus, we have a set of matched pairs, where in each pair, one of them has higher $P_{y}$, the other has higher $P_{o}$, and they differ on the presence or absence of $\sigma$.

What is the effect of this construction? If in each pair, the instance with higher $P_{y}$ is always the one where $I_{\sigma}=1$, it would mean that $\sigma$ always occurs in the instances where the younger author has a higher tendency toward $\sigma$; in other words, we'd be able to perfectly infer the presence or absence of $\sigma$ in each given pair from the relative values of $P_{y}$ and $P_{o}$, with the younger author playing a greater role driving the presence of $\sigma$. If in each pair, the instance with higher $P_{o}$ is always the one where $I_{\sigma}=1$, we would again have perfect prediction with the older author driving the presence of $\sigma$. In general, we say that in a single pair, low experience is dominant if $I_{\sigma}=1$ in the instance with higher $P_{y}$, and high experience is dominant if $I_{\sigma}=1$ in the instance with higher $P_{o}$. If the $I_{\sigma}$ values were assigned at random, we'd expect

\footnotetext{
${ }^{8}$ We also add some additional filters, including a sufficiently high experience for the older author, and at least 10 lifetime papers by the younger author that are not written with the older author.

${ }^{9}$ Since the younger author often has relatively few papers at the time of the fight, we use the set of all papers written by each author (not counting their joint papers) to determine these fractions. This uses information from the future beyond the paper itself, but note that we are not using this for a prediction task, only to determine the relative tendencies of the authors to use the convention over their lifetimes.
} 
low experience and high experience to each be dominant in half the pairs. What do we see in the actual pairs?

We find that in approximately $57 \%$ of the pairs, high experience is dominant, which at the number of pairs we have is significant relative to a random assignment baseline with $p<.001$. Moreover, we can group the pairs into buckets based on $E_{o}-E_{y}$, and perform this analysis on each bucket separately. As we see in the middle panel of Figure 3 , the extent to which high experience is dominant is increasing in the experience difference - the opposite effect from what we saw in the left panel for fights over macro names.

Thus, we have a concrete sense in which older authors are winning visible fights over features of the paper title, even though they are losing invisible fights over macro names.

\subsection{Low visibility fights}

We have now seen the outcomes of two types of fights representing opposite extremes of visibility — fights over macro names, which are essentially invisible to readers; and fights over stylistic conventions in a paper's title, which is extremely visible. Since younger authors tend to win the invisible fights and lose the visible fights in these formulations, it becomes natural to probe the spectrum of possible fights in between these extremes and thus gain more insight into how the outcome of a fight relates to its level of visibility.

This is largely an open question, but here we describe one initial investigation in this direction. Consider the case in which two authors meet to write a paper, and they each use the same macro name but for different bodies. For example, both authors might use \eps, but one uses it to mean \epsilon $(\epsilon)$ while the other uses it to mean \varepsilon $(\varepsilon)$. Whose macro body will end up getting used in the paper they write together? We will call this a macro-body fight, and what's interesting is that it has exactly the structure of our earlier macro-name fights, except with the roles of the name and the body reversed: now the authors arrive with a shared name corresponding to different bodies, and this contention must be resolved. An important contrast, however, is that for many macro bodies, the outcome of this fight will be visible, albeit often at a very low level in the formatting and choice of symbols in the paper. We can therefore think of these as low-visibility fights, and can ask whether younger or older authors will tend to win them.

To explore this question, we need to deal with the fact that not all macro-body fights will have visible effects. Thus we select a small number of very common macro names where the effects of different bodies are generally visible in the paper. Specifically, we use the following names: Iproof, leps and $\backslash$ Re. For these fights we run the same procedure as for macro-name fights, but we swap the roles of the name and body of the macro, and we remove the filter of length 20 , since names and bodies are generally short in this case. With these three macro names we end up with 1092 fight instances. We observe that the young author wins $60 \%$ of the instances, suggesting that the pattern of outcomes is closer to what we saw in the invisible macro-name fights. Grouping the results by the difference in experience, we see in the right panel of Figure 3 that these low-visibility fights follow the same trend as the invisible fights.

\section{DISCUSSION}

Analyzing the competition among conventions has been a methodological challenge, because the substance underly- ing the convention generally changes, at least to some extent, together with the convention itself. We study a setting — macros on the e-print arXiv — where it is possible to fully control for the meaning of the convention (the body of the macro) even as the convention itself (the choice of name) is changing. In the resulting analysis, we focus on two main issues. First, we find that instances in which one macro name convention overtakes another are characterized by young initial users of the convention that ultimately succeeds, together with a transitional phase in which the successful convention spreads to older users. Second, we consider the local, instance-by-instance competition among pairs of authors who must resolve contention over the choice of convention in the process of writing a joint paper. In this type of diffusion through interaction, we find that younger authors tend to win fights (such as for macro names) that do not produce visible consequences, or produce low-visibility consequences, while older authors tend to win fights (such as for titling conventions) that produce highly visible consequences.

Having a clean methodology to study conventions that are synonymous makes possible a number of further directions for research. First, there are other domains where it should be possible to control for the meaning of a convention, for example in repositories of source code where naming conventions can change while the behavior of the code remains constant. It is an interesting question to see whether similar phenomena hold in the dynamics of conventions there. More generally, it is an interesting question to look for additional structure beyond the changeover and fight dynamics presented here in the competition between these conventions. And finally, it is intriguing to consider using the mathematics developed around the theory of neutral variation 20 to begin developing models for the evolution of synonymous conventions over time.

Acknowledgments. We are grateful to Paul Ginsparg for his advice and for his help with the arXiv dataset and to Dan Jurafsky and Justine Zhang for helpful discussions. Very few macro fights were lost during this writeup. This work was supported in part by ARO, Facebook, Google, the Simons Foundation, and a Discovery and Innovation Research Seed Award from Cornell's OVPR.

\section{REFERENCES}

[1] L. A. Adamic and N. Glance. The political blogosphere and the 2004 US election: Divided they blog. In International workshop on link discovery, 2005.

[2] L. A. Adamic, T. M. Lento, and A. T. Fiore. How you met me. In Proc. ICWSM, 2012.

[3] W. B. Arthur. Competing technologies, increasing returns, and lock-in by historical events. Econ.J., 1989.

[4] L. Backstrom, D. Huttenlocher, J. Kleinberg, and X. Lan. Group formation in large social networks: Membership, growth, and evolution. In Proc KDD, 2006.

[5] E. Bakshy, S. Messing, and L. Adamic. Exposure to ideologically diverse news and opinion on Facebook. Science, 52015.

[6] M. Bendersky and D. A. Smith. A dictionary of wisdom and wit: Learning to extract quotable phrases. In Proc. NAACL-HLT, 2012. 
[7] J. Berger and K. L. Milkman. What makes online content viral? Journal of Marketing Research, 2012.

[8] R. S. Burt. Structural holes and good ideas. American Journal of Sociology, 110(2), 2004.

[9] J. Cheng, L. A. Adamic, P. A. Dow, J. M. Kleinberg, and J. Leskovec. Can cascades be predicted? In Proc. $W W W, 2014$.

[10] R. Crane and D. Sornette. Robust dynamic classes revealed by measuring the response function of a social system. In Proc. PNAS, volume 105, 2008.

[11] C. Danescu-Niculescu-Mizil, J. Cheng, J. Kleinberg, and L. Lee. You had me at hello: How phrasing affects memorability. In Proc. ACL, 2012.

[12] C. Danescu-Niculescu-Mizil, R. West, D. Jurafsky, J. Leskovec, and C. Potts. No country for old members: User lifecycle and linguistic change in online communities. In Proc. $W W W, 2013$.

[13] P. Deutschmann. Communication and adoption patterns in an Andean village. Technical report, Prog. Interamericano de Información Popular, 1962.

[14] J. Eisenstein. Phonological factors in social media writing. In Proc. LASM Workshop, 2013.

[15] J. Eisenstein. What to do about bad language on the internet. In Proc. NAACL-HLT, 2013.

[16] M. Gentzkow and J. Shapiro. What drives media slant? Evidence from u.s. daily newspapers. Econometrica, 78(1), 2010.

[17] R. Goel, S. Soni, N. Goyal, J. Paparrizos, H. Wallach, F. Diaz, and J. Eisenstein. The social dynamics of language change in online networks. SocInfo, 2016.

[18] S. Goel, A. Anderson, J. M. Hofman, and D. J. Watts. The structural virality of online diffusion. Management Science, 2016.

[19] W. L. Hamilton, J. Leskovec, and D. Jurafsky. Diachronic word embeddings reveal statistical laws of semantic change. In Proc. ACL, 2016.

[20] M. Kimura. The Neutral Theory of Molecular Evolution. Cambridge University Press, 1983.

[21] F. Kooti, H. Yang, M. Cha, K. P. Gummadi, and W. A. Mason. The emergence of conventions in online social networks. In Proc. ICWSM, 2012.

[22] D. Krackhardt. Organizational viscosity and the diffusion of innovations. J. Math. Soc., 1997.

[23] W. Labov, I. Rosenfelder, and J. Fruehwald. One hundred years of sound change in philadelphia: Linear incrementation, reversal, and reanalysis. Language, 2013.

[24] W. L. Labov. The Social Stratification of English in New York City. Center for Applied Linguistics, 1966.

[25] W. S. E. Lam. Language Socialization in Online Communities. Springer US, 2008.

[26] J. Leskovec, L. Adamic, and B. Huberman. The dynamics of viral marketing. ACM TWEB, 2007.

[27] D. Liben-Nowell and J. Kleinberg. Tracing information flow on a global scale using Internet chain-letter data. In Proc. PNAS, Mar. 2008.

[28] A. Livne, M. P. Simmons, E. Adar, and L. A. Adamic. The party is over here: Structure and content in the 2010 election. In Proc. ICWSM, 2011.
[29] S. K. Maity, R. Saraf, and A. Mukherjee. \# bieber+\# blast=\# bieberblast: Early prediction of popular hashtag compounds. In Proc. CSCW, 2016.

[30] M. W. McLaughlin. The Rand change agent study revisited: Macro perspectives and micro realities. Educational Researcher, 19(9), 1990.

[31] E. Omodei, T. Poibeau, and J.-P. Cointet. Multi-level modeling of quotation families morphogenesis. In SocialCom. IEEE, 2012.

[32] C. Peersman, W. Daelemans, R. Vandekerckhove, B. Vandekerckhove, and L. Van Vaerenbergh. The effects of age, gender and region on non-standard linguistic variation in online social networks. arXiv preprint arXiv:1601.02431, 2016.

[33] F. Perek. Vector spaces for historical linguistics: Using distributional semantics to study syntactic productivity in diachrony. In Proc. $A C L, 2014$.

[34] E. Rogers. Characteristics of agricultural innovators and other adopter categories. Technical Report 882, Agricultural Experimental Station, Wooster OH, 1961.

[35] E. Rogers. Diffusion of Innovations. Free Press, 1995.

[36] D. M. Romero, B. Meeder, and J. Kleinberg. Differences in the mechanics of information diffusion across topics: Idioms, political hashtags, and complex contagion on Twitter. In Proc. WWW, 2011.

[37] D. M. Romero, C. Tan, and J. Ugander. On the interplay between social and topical structure. In Proc. ICWSM, 2013.

[38] R. Rotabi and J. Kleinberg. The status gradient of trends in social media. In Proc. ICWSM, 2016.

[39] M. Salganik, P. Dodds, and D. Watts. Experimental study of inequality and unpredictability in an artificial cultural market. Science, 311:854-856, 2006.

[40] G. Simmel. The Sociology of Georg Simmel. Free Press (translated by Kurt H. Wolf), 1908.

[41] N. Tahmasebi, T. Risse, and S. Dietze. Towards automatic language evolution tracking, a study on word sense tracking. In Workshop on Knowledge Evolution and Ontology Dynamics, 2011.

[42] O. Tsur and A. Rappoport. What's in a hashtag?: content based prediction of the spread of ideas in microblogging communities. In Proc. WSDM, 2012.

[43] O. Tsur and A. Rappoport. Don't let me be \#misunderstood: Linguistically motivated algorithm for predicting the popularity of textual memes. In Proc. ICWSM, 2015.

[44] J. Ugander, L. Backstrom, C. Marlow, and J. Kleinberg. Structural diversity in social contagion. In Proc. PNAS, 2012.

[45] T. Valente. Network interventions. Science, 2012.

[46] D. Willer. Network Exchange Theory. Praeger, 1999.

[47] H. P. Young. The economics of convention. Journal of Economic Perspectives, 10(2):105-122, Spring 1996. 